\title{
Catching nucleosomes in action
}

Techniques such as chromatin immunoprecipitation have enabled great advances through providing 'snapshots' of the epigenome, but it remains technically difficult to study the dynamics of nucleosome turnover. A new method that directly assays native histone turnover shows great promise and has already challenged models of epigenetic maintenance.

Previously, nucleosome turnover has been studied either by mapping the histone variant $\mathrm{H} 3.3$, which is deposited independently of DNA replication, or by using transgenes in yeast. In their new approach, Deal and colleagues assayed the kinetics of native histones by

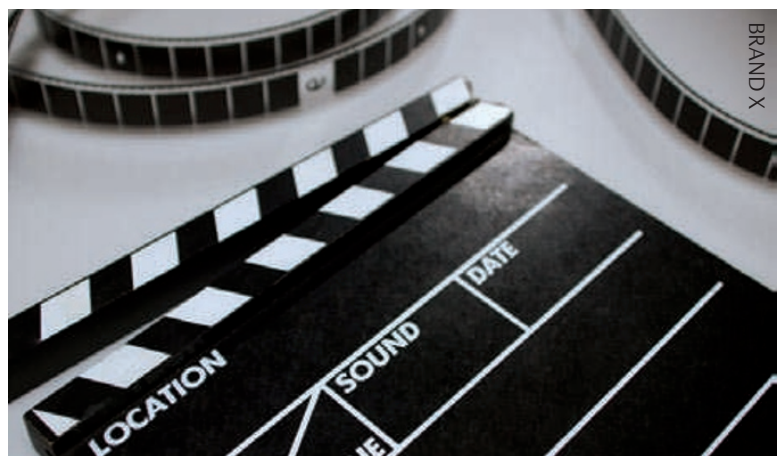

pulsing Drosophila melanogaster S2 cells with the methionine (Met) surrogate azidohomoalanine (Aha); the surrogate amino acid is incorporated into histones that are being translated. The authors then couple biotin to Aha, break up the chromatin into mononucleosomes and use streptavidin beads to affinity purify nucleosomes containing newly synthesized histones. They hybridized the nucleosomal DNA to a highdensity tiling microarray to create a map of nucleosomes that have turned over during the pulse. The strategy is called CATCH-IT (covalent attachment of tags to capture histones and identify turnover) and should be applicable across species.

The CATCH-IT landscapes show good correspondence with patterns of H3.3 but with greater definition of chromatin features. Also, turnover kinetics can be measured by varying the length of the Aha pulse. Deal and colleagues examined turnover at different genomic locations and showed that nucleosomes in active genes and at epigenetic regulatory elements such as binding sites for polycomb or trithorax group proteins - would be replaced multiple times during a cell cycle of $\sim 20$ hours.

These findings have important implications for understanding the maintenance of epigenetic information. Histones that have been modified (by enzymes such as the polycomb protein enhancer of zeste) turn over faster than the cell cycle, which suggests that the modifications themselves are not transmitting epigenetic information. The authors suggest that it might be alteration in DNA accessibility through regulated nucleosome turnover that maintains active or silent gene expression states. Further data from the CATCH-IT method could test this proposal and may lead to new models of epigenome function.

Mary Muers

ORIGINAL RESEARCH PAPER Deal, R. B., Henikoff, J. G. \& Henikoff, S. Genome-wide kinetics of nucleosome turnover determined by metabolic labeling of histones. Science $\mathbf{3 2 8}$, 1161-1164 (2010)

FURTHER READING Henikoff, S. Nucleosome destabilization in the epigenetic regulation of gene expression. Nature Rev. Genet. 9, 15-26 (2008) | Margueron R. \& Reinberg, D. Chromatin structure and the inheritance of epigenetic information. Nature Rev. Genet. 11, 285-296 (2010) 